\title{
Ventricular Tachycardia in Neonates: A Report of Two Cases and Review of the Literature
}

\author{
Yoo Ra Jung, Sun Hyang Lee, Ga Young Park, Sung Shin Kim \\ Department of Pediatrics, Soonchunhyang University Bucheon Hospital, Soonchunhyang University College of Medicine, Bucheon, Korea
}

\begin{abstract}
In the case of hemodynamically stable broad complex tachycardia, it is essential to evaluate the causes and to diagnose correctly in the selection of appropriate management and drugs. We report two neonates diagnosed with idiopathic ventricular tachycardia, which is extraordinarily rare. One presented with idiopathic fascicular ventricular tachycardia (right bundle branch block pattern with a superior axis), and the other presented with right ventricular outflow tract ventricular tachycardia (left bundle branch block pattern with an inferior axis). These two forms are representative of benign ventricular tachycardia. No features of cardiovascular shock were observed. While the recommended initial drug treatments are different, the conditions were well controlled by propranolol without the development of any adverse events. There was no recurrence of arrhythmia for several months in the outpatient clinic.
\end{abstract}

Keywords: Arrhythmia; Neonate; Propranolol; Ventricular tachycardia

\section{INTRODUCTION}

Among the types of arrhythmia that occur in neonates, ventricular tachycardia (VT) is very rare, resulting in a lack of known therapeutic drugs and clinical outcomes. Hemodynamically unstable VT must be immediately managed in accordance with the pediatric advanced resuscitation guidelines. On the other hand, when hemodynamically stable VT occurs, careful selection of appropriate acute management and maintenance drugs among the limited drugs available for neonates is required. In this report, we describe the representative types of idiopathic VT in neonates and appropriate drugs based on clinical manifestations.

\section{CASE REPORT}

\section{Ethical statement}

This retrospective chart review study involving human participants was in accordance with the ethical standards of the institutional and national research committee and with the 1964 Helsin- ki Declaration and its later amendments or comparable ethical standards. This study was approved by the Institutional Review Board of Soonchunhyang University Bucheon Hospital (SCHBC 2020-04-015). Written informed consent by the patient was waived due to a retrospective nature of our study.

\section{Case 1}

A 9-day-old term neonate was presented to a hospital for suspected arrhythmia. On admission to the neonatal intensive care unit (NICU), the neonate was found to exhibit frequent ventricular premature beats and nonsustained VT with a heart rate of 170 beats per minute (bpm) on an electrocardiographic monitor. Dyspnea, tachypnea, or other signs of cardiovascular collapse were not observed. The laboratory tests were non-specific. The echocardiogram showed a small muscular ventricular septal defect (Fig. 1) and normal cardiac function. Twelve-lead electrocardiography (ECG) demonstrated broad complex tachycardia at a rate of 170 bpm with a superior axis and right bundle branch block (RBBB)like pattern (Fig. 2). He was diagnosed with fascicular VT. Oral 
propranolol ( $0.5 \mathrm{mg} / \mathrm{kg} /$ dose 3 times a day) was administered initially. The oral maintenance dose was increased to $1 \mathrm{mg} / \mathrm{kg} / \mathrm{dose} 3$ times a day due to an episode of sustained VT. His blood pressure remained stable throughout. The neonate was discharged after a few days of observation without the recurrence of tachycardia (Fig. 3).

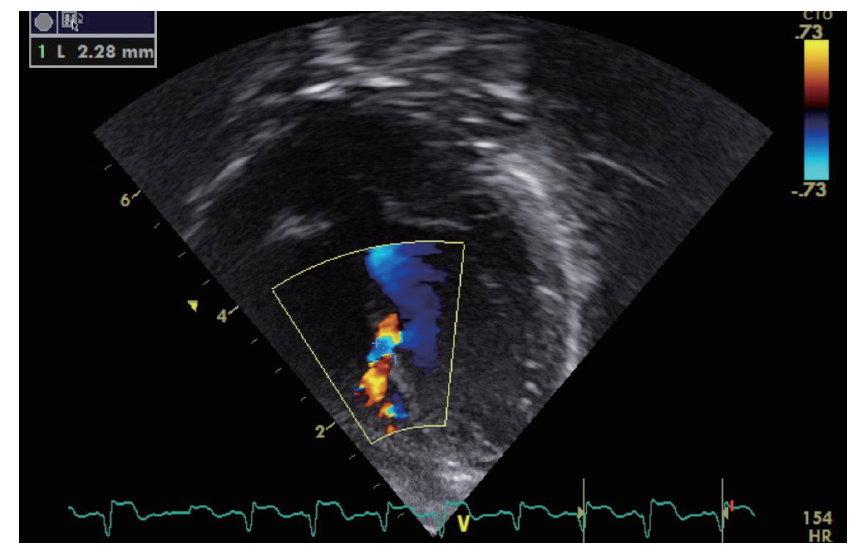

Fig. 1. Transthoracic echocardiography demonstrates a small mid-muscular ventricular septal defect with left-to-right shunt by color doppler imaging (marked by crosshairs).

\section{Case 2}

A 3-day-old term neonate was referred to a hospital for suspected arrhythmia and a short-term history of decreased activity. On admission to the NICU, the neonate was found to exhibit apnea with desaturation and nonsustained VT (200 bpm) on an electrocardiographic monitor. There were no signs of cardiovascular collapse. The laboratory tests were non-specific. The echocardiogram showed a small atrial septal defect (Fig. 4) and normal cardiac function. Twelve-lead ECG demonstrated broad complex tachycardia at a rate of $200 \mathrm{bpm}$ with an inferior axis, left bundle branch block (LBBB)-like pattern, and atrioventricular dissociation presumed to be right ventricular outflow tract VT (Fig. 5). An infusion of $50 \mathrm{mcg} / \mathrm{kg} / \mathrm{min}$ of esmolol and free-flow oxygen were administered initially. VT did not recur after the drug dose was increased to $150 \mathrm{mcg} / \mathrm{kg} / \mathrm{min}$ on the second day of hospitalization. After several days, the oral maintenance dose was controlled by $1.2 \mathrm{mg} / \mathrm{kg} /$ day, but on the 8th day of hospitalization, the drug dose was gradually increased due to the recurrence of sustained VT. By increasing the drug dose, respiratory depression, poor feeding, and bradycardia were observed, requiring dose control. The oral

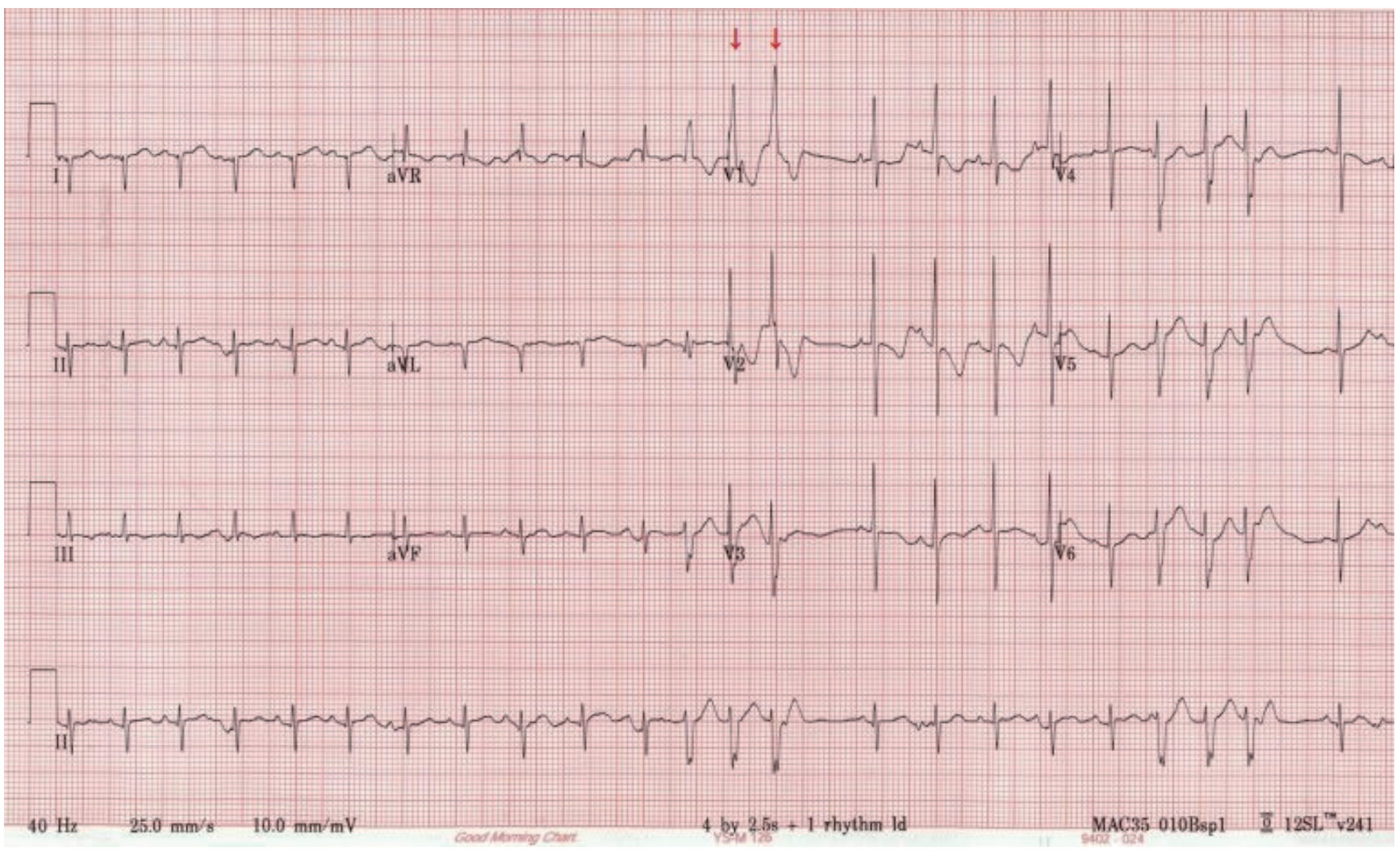

Fig. 2. Idiopathic fascicular ventricular tachycardia. Note the wide QRS complex with a right bundle branch block pattern (arrows) and superior axis. A high ventricular rate of 170 beats per minute is noted. 


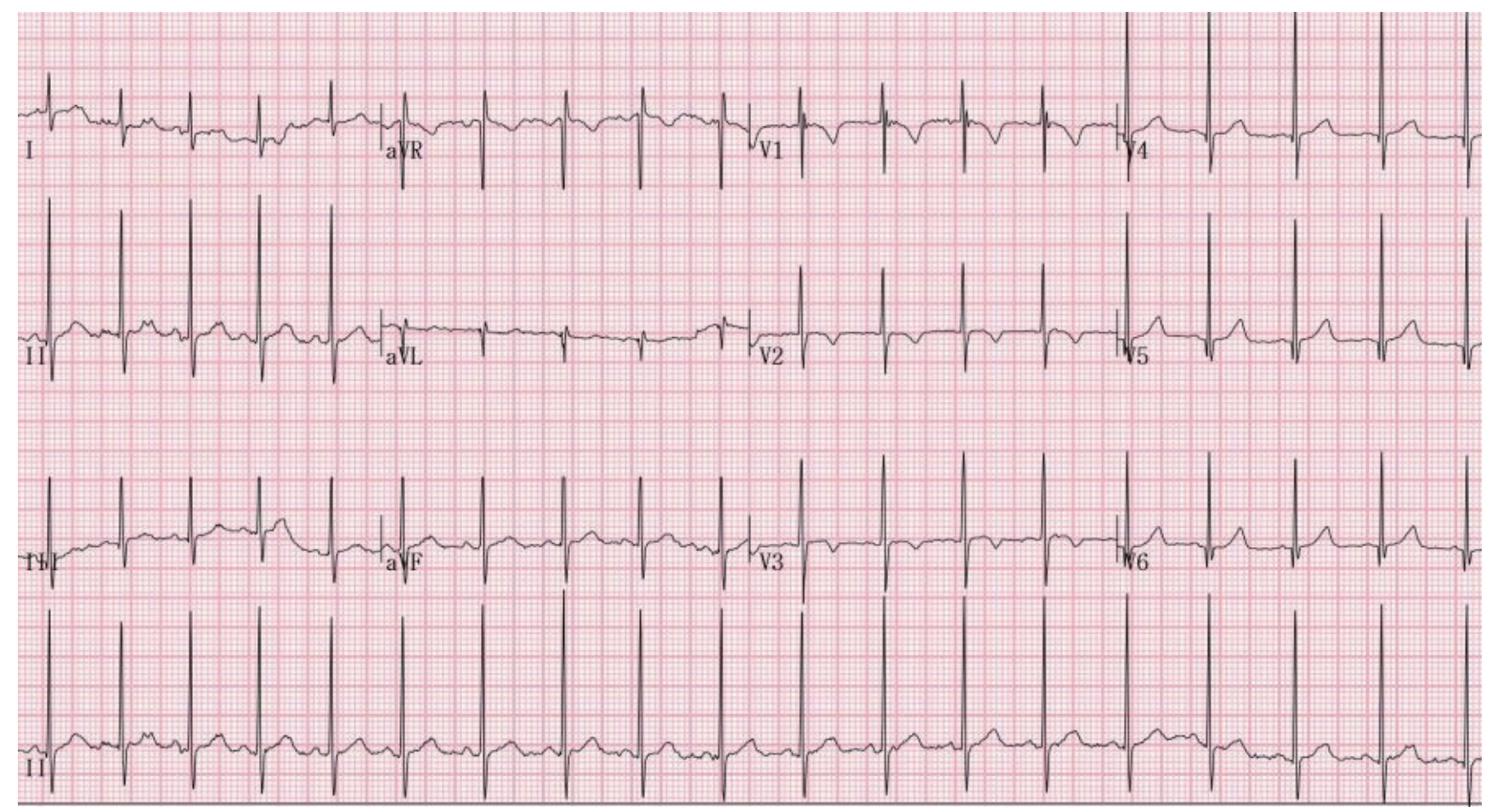

$25 \mathrm{~mm} / \mathrm{s} \quad 10 \mathrm{~mm} / \mathrm{mV} \quad 16-150 \mathrm{~Hz} \quad 60 \mathrm{~Hz}$

Fig. 3. Oral propranolol administration converted ventricular tachycardia to a normal sinus rhythm.

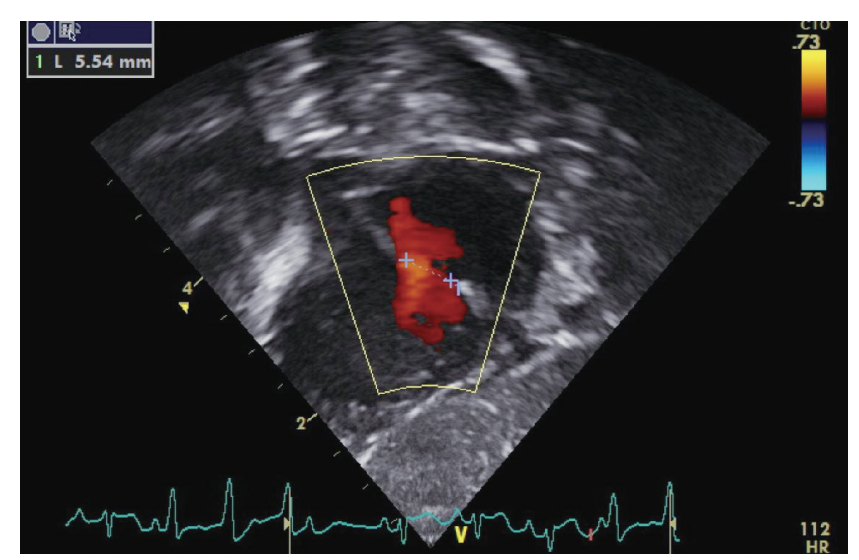

Fig. 4. Transthoracic echocardiography demonstrates a small secundum atrial septal defect with left-to-right shunt by color doppler imaging (marked by crosshair).

maintenance dose was increased to $12 \mathrm{mg} / \mathrm{kg} /$ day 4 times a day, and the neonate was discharged without the recurrence of tachycardia or the development of adverse events (Fig. 6).

\section{DISCUSSION}

Among the types of arrhythmia that occur in neonates, VT is very rare, resulting in a lack of known therapeutic drugs and clini- cal outcomes. Hemodynamically unstable VT must be immediately managed in accordance with the pediatric advanced resuscitation guidelines. On the other hand, when hemodynamically stable VT occurs, careful selection of appropriate acute management and maintenance drugs among the limited drugs available for neonates is required. Among cases of VT, idiopathic VT is usually known to not require treatment with a benign, self-limited course $[1,2]$. However, there are reports of possible sudden death in the case of idiopathic VT, thus, preventative maintenance treatment is needed until 1 year of age [2,3]. VT is defined as three or more consecutive premature ventricular contractions (PVCs) at a rate faster than $120 \mathrm{bpm}$ or $20 \%$ greater than the preceding sinus rhythm and has a wide QRS complex with atrioventricular dissociation during the tachycardia. VT at a rate less than $20 \%$ greater than the sinus rhythm is termed an accelerated idioventricular rhythm. Sustained VT is defined as three or more consecutive PVCs lasting for at least 30 seconds [4]. Idiopathic VT is defined as VT without identified underlying causes such as myocarditis, cardiac tumors, congenital heart disease, hereditary cardiomyopathy, long QT syndrome, and electrolyte abnormalities. There are two benign forms of idiopathic VT according to the bundle branch block pattern of QRS morphology that suggests the origin of VT: 


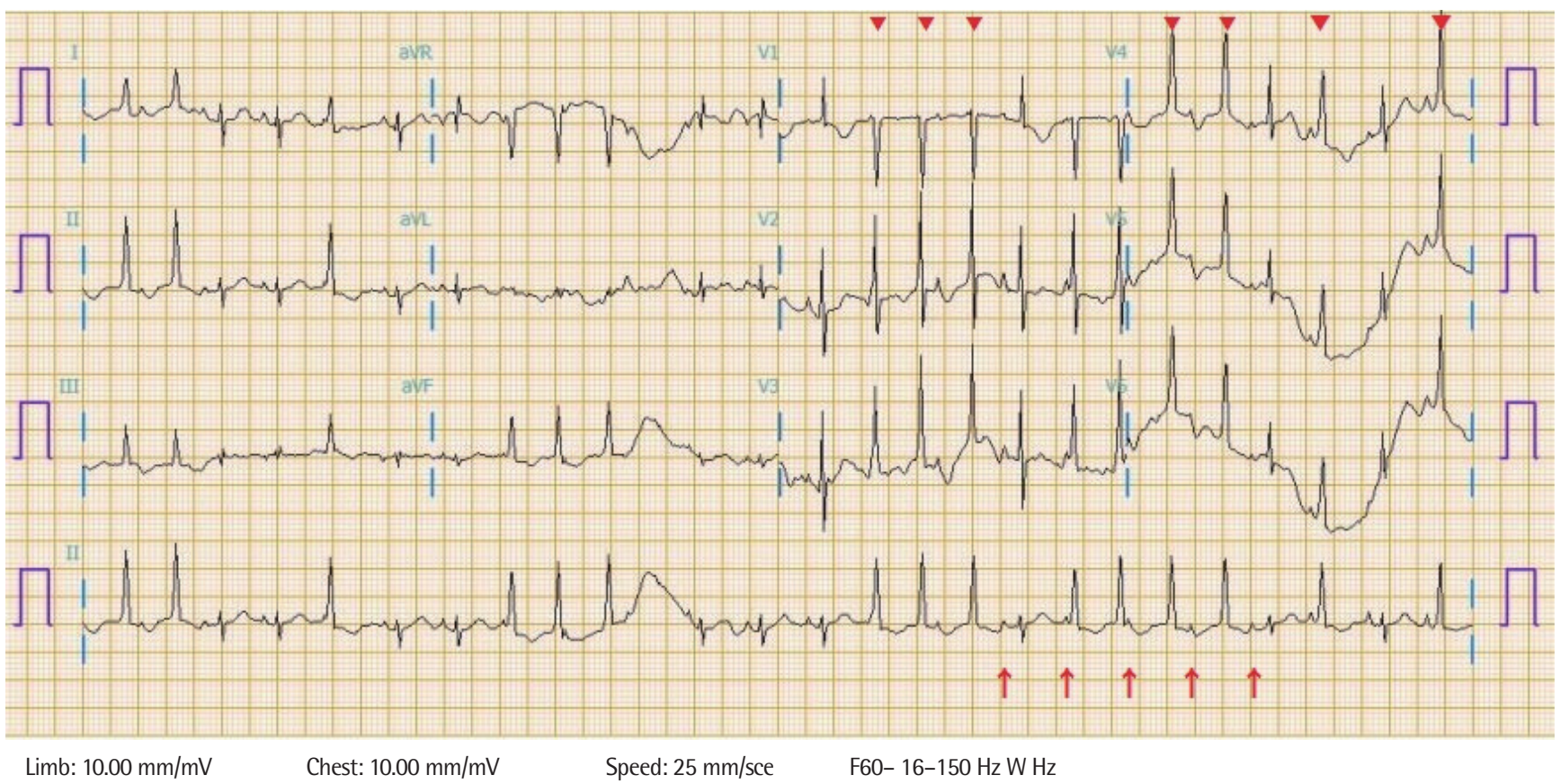

Fig. 5. Right ventricular outflow tract ventricular tachycardia. Note the wide QRS complex with a left bundle branch block pattern (arrowheads) and inferior axis. A high ventricular rate of 200 beats per minute and dissociated p waves (arrows) are characteristic of ventricular tachycardia.

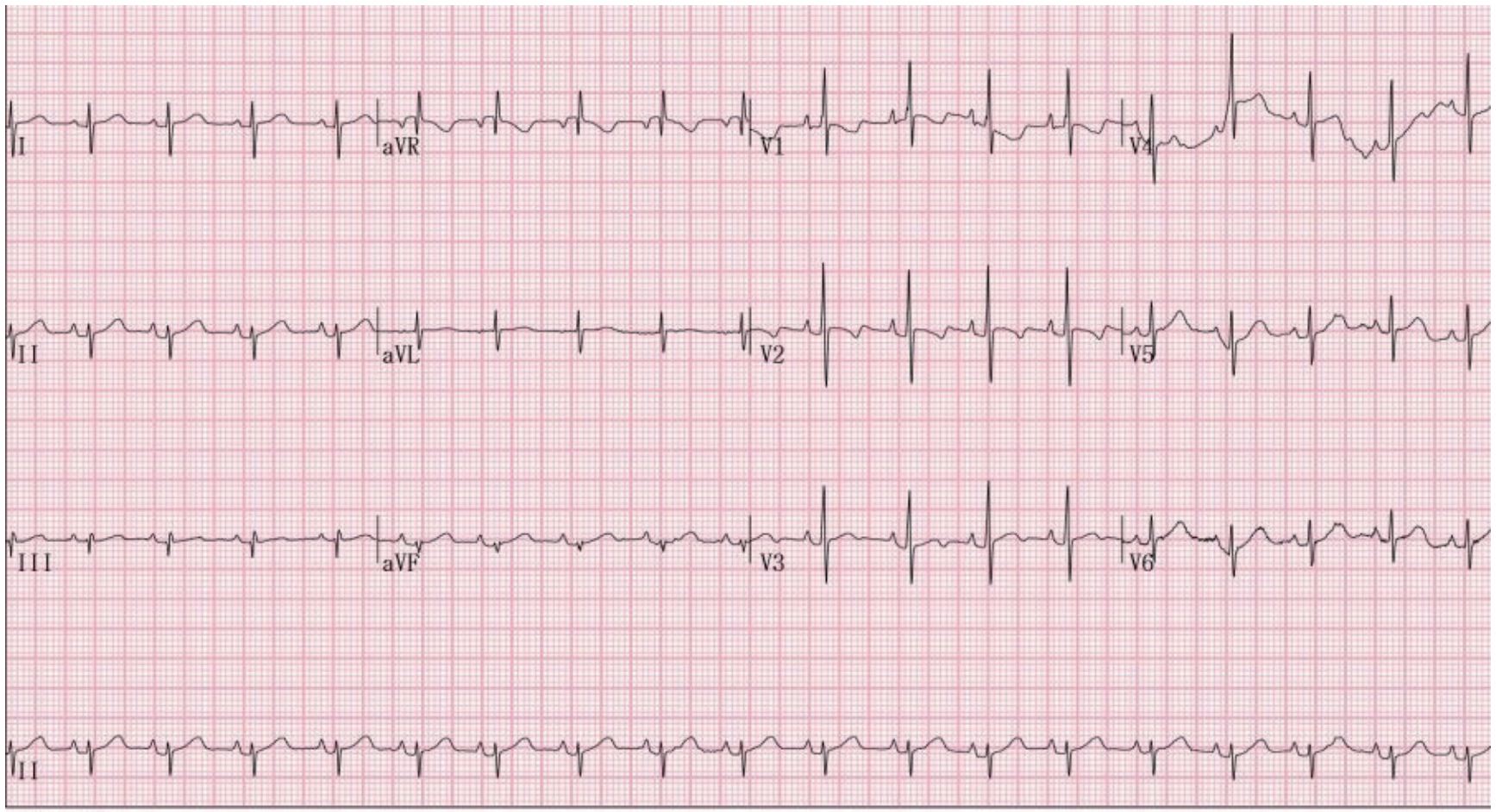

$25 \mathrm{~mm} / \mathrm{s} \quad 10 \mathrm{~mm} / \mathrm{mV} \quad 16-150 \mathrm{~Hz} \quad 60 \mathrm{~Hz}$

Fig. 6. Intravenous esmolol infusion and oral propranolol administration converted ventricular tachycardia to a normal sinus rhythm.

right ventricular outflow tract VT (LBBB pattern with an inferior axis) and idiopathic fascicular VT (RBBB pattern with a superior axis) [5]. For patients with idiopathic fascicular VT, verapamil is the first-choice antiarrhythmic drug. In addition, there are several 
reports of successful conversion of fascicular VT in young infants with intravenous verapamil [6]. However, intravenous administration of verapamil for the treatment of tachyarrhythmia is contraindicated in infants younger than 1 year of age because of the proven risk of hemodynamic collapse and even death. In cases of right ventricular outflow tract VT, beta-blockers are the firstchoice drugs.

In this report, we describe the representative types of idiopathic VT in neonates and appropriate drugs based on clinical manifestations. VT in both patients was well controlled with beta-blockers, and the patients were discharged from the hospital with the prescription of Hemangiol (propranolol hydrochloride $3.75 \mathrm{mg}$ / mL, oral solution; Pierre Fabre Australia Pty Ltd., North Sydney, Australia) for precise drug dose control according to their low body weights. There was no recurrence of arrhythmia for several months in the outpatient clinic. We believe that the treatment of beta-blockers is safe and successful in cases of hemodynamically stable idiopathic VT in neonates. Long-term follow-up is required for recurrence of arrhythmia after discontinuation of the drugs.

\section{CONFLICT OF INTEREST}

No potential conflict of interest relevant to this article was reported.

\section{ORCID}

$\begin{array}{ll}\text { Yoo Ra Jung } & \text { https://orcid.org/0000-0002-5474-5601 } \\ \text { Sun Hyang Lee } & \text { https://orcid.org/0000-0001-7873-8409 } \\ \text { Ga Young Park } & \text { https://orcid.org/0000-0002-9095-6457 } \\ \text { Sung Shin Kim } & \text { https://orcid.org/0000-0001-9724-3006 }\end{array}$

\section{AUTHOR CONTRIBUTIONS}

Conception or design: SHL, GYP, SSK; acquisition, analysis, or interpretation of data: YRJ, SHL; drafting the work or revising: YRJ, SHL; and final approval of the manuscript: YRJ, SHL, GYP, SSK.

\section{REFERENCES}

1. MacLellan-Tobert SG, Porter CJ. Accelerated idioventricular rhythm: a benign arrhythmia in childhood. Pediatrics 1995;96(1 Pt 1):122-5.

2. Davis AM, Gow RM, McCrindle BW, Hamilton RM. Clinical spectrum, therapeutic management, and follow-up of ventricular tachycardia in infants and young children. Am Heart J 1996;131:186-91.

3. Gillette PC. Ventricular tachycardia and accelerated ventricular rhythm presenting in the first month of life. Am J Cardiol 1991;68:840-1.

4. Levin MD, Stephens P, Tanel RE, Vetter VL, Rhodes LA. Ventricular tachycardia in infants with structurally normal heart: a benign disorder. Cardiol Young 2010;20:641-7.

5. Ban JE. Neonatal arrhythmias: diagnosis, treatment, and clinical outcome. Korean J Pediatr 2017;60:344-52.

6. Kehr J, Binfield A, Maxwell F, Hornung T, Skinner JR. Fascicular tachycardia in infancy and the use of verapamil: a case series and literature review. Arch Dis Child 2019;104:789-92. 